\title{
Niemann Pick C: First Case in a Canadian Nakoda Nation Child
}

\author{
Aneal Khan, Colleen Curtis, Harvey B. Sarnat, Alfredo Pinto-Rojas, Khemissa Bejaoui, \\ Xing-Chang Wei, Robin Casey
}

Can J Neurol Sci. 2014; 41: 518-521

Niemann Pick disease type C (NPC) is a neurodegenerative disorder that classically presents in children with hepatosplenomegaly, liver abnormalities (such as jaundice and coagulopathy) and developmental delay ${ }^{1,2}$. Niemann Pick type $\mathrm{C}$ is an autosomal recessive disease caused mostly commonly by mutations in the NPC1 gene and less frequently by mutations in the NPC2 gene $^{3}$. The underlying pathophysiology of NPC involves abnormal trafficking of cholesterol from late endosomes / lysosomes after low density lipoprotein-mediated uptake $^{4}$. The diagnosis is currently established by either gene sequencing or cultured skin fibroblast studies showing decreased cholesterol esterification and increased filipin staining ${ }^{5}$. We find this case to be important for the following reasons: 1 . to provide knowledge of a novel variant present in the Stoney Nakoda Nation, 2. to show that NPC can be present in and obscured by an overlying Epstein-Barr Virus (EBV) infection and 3. that the finding of a non-specific mitochondrial cytopathy in a patient with seizures and chronic encephalopathy may be a secondary feature of underlying NPC.

The Stoney Nakoda Nation originate from the larger Sioux nation, but migrated north and west to the North American prairie and Rocky mountains. Through interaction with other First Nations, and treaties with the Canadian government, they have come to reside in southern Alberta. There is no previous report of NPC in a Stoney Nakoda Nation child.

\section{Clinical Presentation}

The patient had a healthy perinatal course and was described as normal through infancy. At 15 months-of-age, she presented to our hospital with hepatosplenomegaly, tonsillar enlargement, mild developmental delay and hypotonia. Initial investigations showed a positive IgM titre to EBV and EBV capsid antigen (CA) IgG (capsid antigen immunoglobulin G) was negative. On repeat serology two months later, EBV CA IgM was indeterminate and EBV CA IgG was positive, consistent with a diagnosis of acute EBV infection. At re-evaluation at 33 monthsof-age, she had persistence of hepatosplenomegaly, resolution of tonsillar enlargement, but had developed neurologic deterioration, central hypotonia, spasticity of her upper and lower extremities and hyper-reflexia. A brain magnetic resonance image (MRI) showed non-specific symmetric abnormal T2 signal intensity involving the deep white matter of the cerebral hemispheres and normal diffusion. Nerve conduction studies showed reduced conduction velocity in the motor nerves. The left median motor velocity was $31.7 \mathrm{~m} / \mathrm{s}$ (reference $>50 \mathrm{~m} / \mathrm{s}$ ), left peroneal motor velocity $34.9 \mathrm{~m} / \mathrm{s}$ (reference $>38 \mathrm{~m} / \mathrm{s}$ ) and left ulnar motor velocity $32.1 \mathrm{~ms} /$ (reference $>53 \mathrm{~m} / \mathrm{s}$ ) (J. Mah, Alberta Children's Hospital). Electromyography demonstrated increased insertional activity and runs of positive sharp waves in the left deltoid, suspicious for myopathic changes. She had a persistent elevation in aspartate aminotransferase (AST) (57-93, normal 1-35 U/L), lactate dehydrogenase (LDH) (330-428, normal 125-320 U/L) units, and INR (1.1-1.4, normal 0.9-1.1) but normal alanine aminotransferase (ALT), alkaline phosphatase, direct and indirect bilirubin. Due to the rapid progression of the underlying disease at this stage, the family consented to selective investigations under a single general anesthetic: a muscle biopsy, skin biopsy and nerve biopsy. The skin biopsy from the lateral aspect of the thigh showed abnormal lysosomes in cutaneous nerves and mitochondrial ultrastructural abnormalities [Figure 1A-D]. The vastus lateralis muscle biopsy showed histochemical and ultrastructural evidence of a mitochondrial cytopathy [Figure 2A-C]. Mitochondrial electron transport chain analysis revealed decreased complex III specific activity of 0.4 $\mathrm{nmol} / \mathrm{min} / \mathrm{mg}$ protein (control 3-35) and complex V of $20 \mathrm{nmol} /$ $\mathrm{min} / \mathrm{mg}$ protein (control 42-160) but normal complex I, II, IV and a citrate synthase control of $63 \mathrm{nmol} / \mathrm{min} / \mathrm{mg}$ protein (control 44-160) (J. Marin, The Molecular Cardiology and Neuromuscular Institute Inc, Highland Park, NJ). A chest X-ray showed a diffuse interstitial reticulonodular pattern. The patient died shortly thereafter from respiratory failure. Autopsy confirmed the finding of foam cells in the liver and bone marrow [Figure 3A-B]. Skin fibroblasts showed $0 \%$ cholesterol esterification compared to controls and abnormal filipin staining (D. Matern, Mayo Medical Laboratories, Rochester, MN).

Sequencing of the NPC1 gene showed the patient was homozygous for a sequence variant defined as c.467T $>\mathrm{G}$, and predicted to result in the amino acid substitution p.Met156Arg.

From Medical Genetics and Pediatrics (AK, RC), Pathology (APR), Neuropathology and Clinical Neurosciences (HBS), Diagnostic Imaging (XCW), Alberta Children's Hospital (CC), University of Calgary (AK, RC), Calgary, Alberta, Canada;

PreventionGenetics (KB), Marshfield, Wisconsin, USA.

Received February 26, 2013. Final Revisions Submitted February 6, 2014. Correspondence to: Aneal Khan, Medical Genetics and Pediatrics, University of Calgary, Alberta Children's Hospital, 2888 Shaganappi Trail NW, Calgary, Alberta, T3B 6A8, Canada. Email: khaa@ucalgary.ca. 


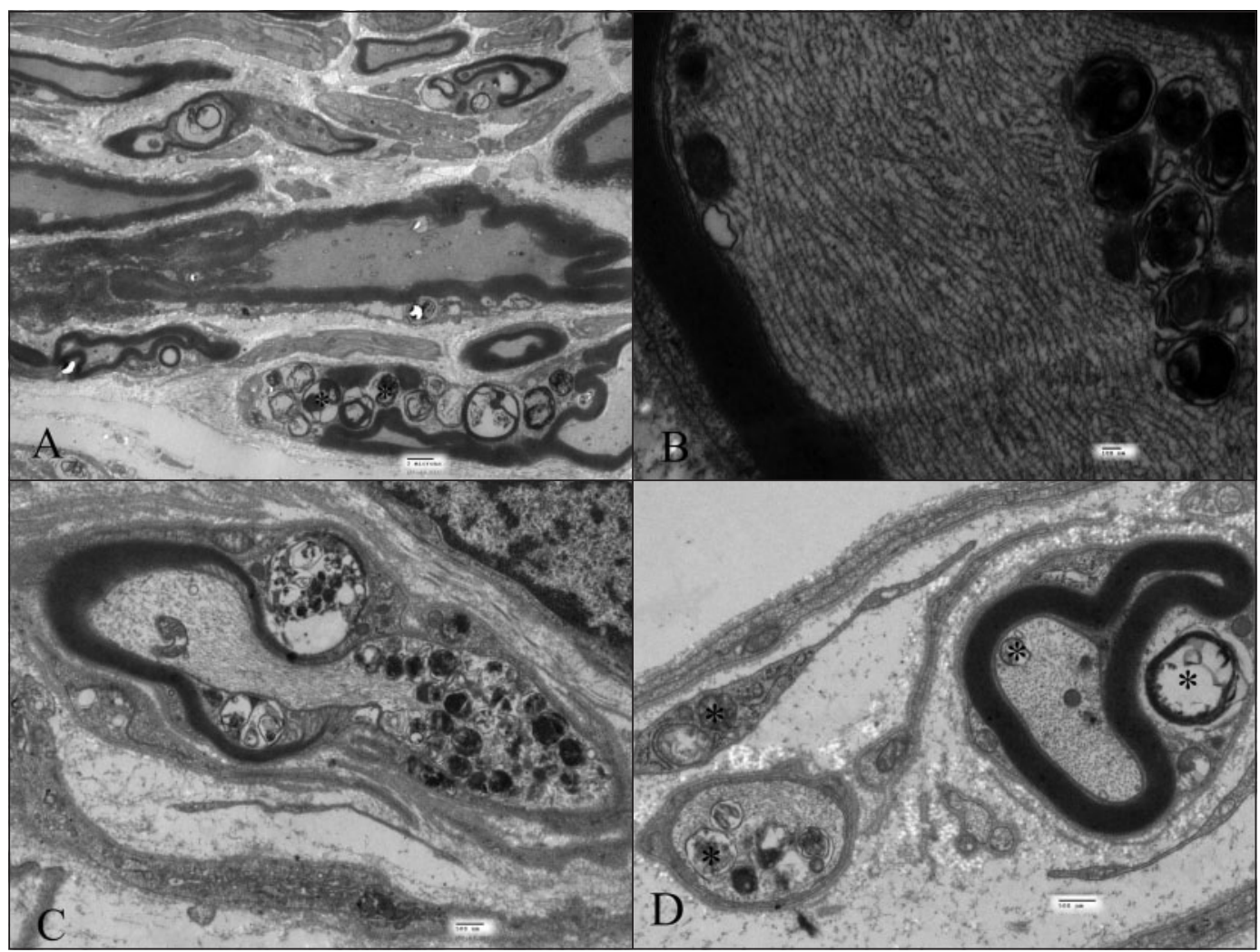

Figure 1: Transmission electron microscopy of cutaneous nerve in skin biopsy at age 14 months. (A) Schwann cell cytoplasm is filled with large lysosomes filled with amorphous debris, but myelinated and unmyelinated axons and myelin sheaths remain well preserved. (B) A myelinated axon exhibits good preservation of its myelin sheath, neurofilaments and microtubules, despite an aggregate of lysosomes within the axoplasm. (C) At a node of Ranvier, a large cluster of lysosomes is seen in the unmyelinated portion and the axoplasm is well preserved in the myelinated portion of the axon, including a large mitochondrion with well-formed cristae; the myelin sheath and transitional zone to the unmyelinated part of the axon are not altered. A large lysosome also is seen in a Schwann cell. The increased interperiodic distance between turns of myelin at the transition is an expected feature of the nodes of Ranvier. (D) Multiple lysosomes (*) are demonstrated in myelinated and unmyelinated axons, in Schwann cells and in a fibroblast (in the upper part of the figure). The fibroblast contains well-formed granular endoplasmic reticulum and lacks a basal lamina (normal feature of fibroblasts).

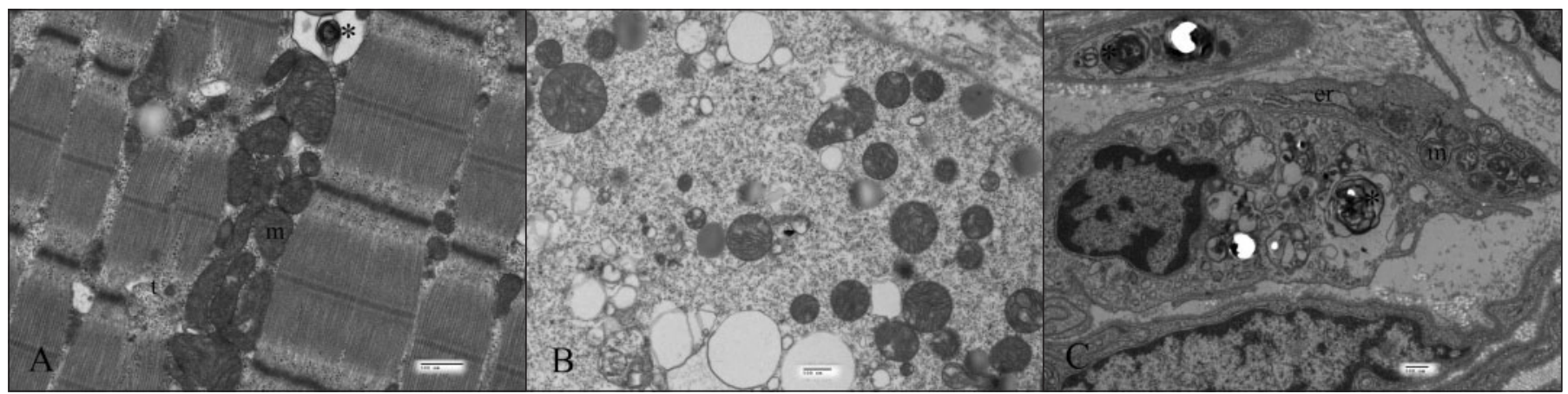

Figure 2: Transmission electron microscopy of a muscle biopsy at age 14 months. (A) Myofibrils are well organized into repeating sarcomeres with well-formed Z- and M-bands, as seen in longitudinal section. Clusters of intermyofibrillar mitochondria $(m)$ have preserved cristae without stacking, whorling or formation of paracrystallin bodies. A probable lysosome (*) also is seen in the sarcoplasm. (B) The subsarcolemmal region of a myofibre contains multiple pleomorphic mitochondria without frank whorling of cristae, but in which cristae often have an abnormal form or parallel clustering. (C) Perimysial fibroblasts are filled with large lysosomes $(*)$ containing amorphous débris. Normal granular endoplasmic reticulum (er) is seen. Mitochondria $(m)$ are swollen and cristae are fragmented. 


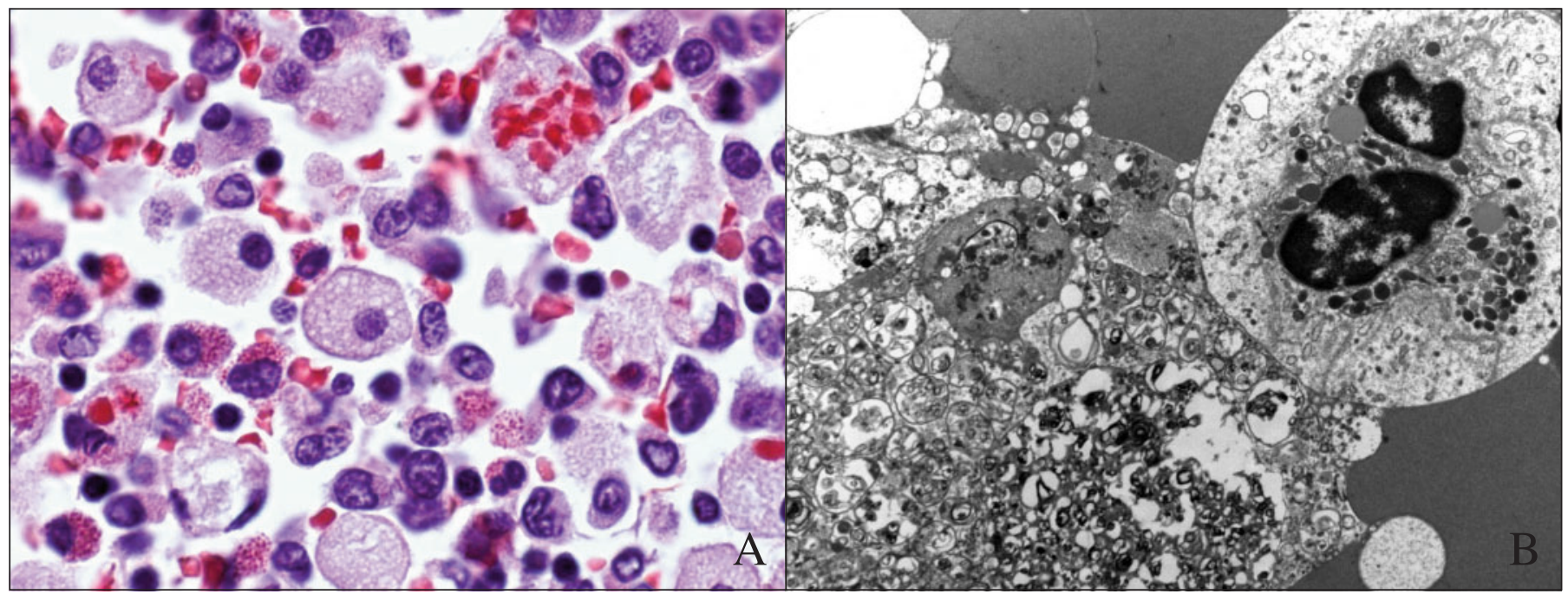

Figure 3: (A) Bone marrow infiltrated by foamy histiocytes with numerous small clear vacuoles in the cytoplasm (hematoxilin eosin 40X). (B) Ultrastructure of the cytoplasm of a histiocyte showing numerous membranous lamellar inclusions, pleomorphic bodies with dense and lucent zones and dense granules (uranyl acetate, lead citrate 2500X).

This particular variant was not reported previously in the literature or any of the public databases, indicating that it is rare.

The p.Met156 residue of the NPC1 protein has been well conserved during evolution, and the amino acid substitution prediction tools of Alamut predicted the change to be deleterious (Alamut v2.0 Interactive Biosoftware, http://www.interactivebiosoftware.com/). Based on the available information, we suspect that the novel c.467T $>\mathrm{G}$ variant is the cause of NPC in this patient. Both parents originate from Stoney Nakoda Nation ancestry, but we were not able to collect DNA on the parents for further evaluation.

\section{DisCUSSION}

In a child presenting with multi-system disease, the consideration of an underlying rare disease, such as NPC, can be overlooked. In this case, the co-existence of EBV infection and the finding of mitochondrial cytopathy obscured the underlying diagnosis. However, clinical suspicion for NPC was aided by the characteristic pattern of pulmonary lipoid pneumonia ${ }^{6}$, which is seen in virtually all patients with advanced NPC due to NPC2 mutations, but also due to NPCl mutations, and the lysosomal histopathology on nerve and skin biopsy ${ }^{6}$. Confirmatory studies from skin fibroblasts and mutation analysis returned only after her death. Secondary mitochondrial pathological changes can be seen in $\mathrm{NPC}^{7}$ and are likely due to the essential role of cholesterol in mitochondrial membranes. Cholesterol is metabolized to pregnanolone in mitochondria for the production of neuronal steroids in myelinated axons ${ }^{8}$. In myelinated axons from cutaneous nerves on the skin biopsy, our case shows lysosomal accumulation. The muscle biopsy, however, shows that mitochondria in muscle can also be affected and this may contribute to the neuromuscular deterioration seen in some patients. Hepatomegaly can be a common feature in children with mitochondrial disease but splenomegaly would be an unusual finding. Abnormal mitochondrial structure and function in neurons was reported in a mouse model of NPC1 ${ }^{9}$. In the cells described by Yu et al, there was an increase in cholesterol in the mitochondrial membrane, decreased mitochondrial complex V activity and a decrease in mitochondrial adenosine triphosphate (ATP) production. In their model, the addition of cholesterol to mitochondrial membranes of wild type mouse samples reproduced the impaired ATP formation seen in mutant mouse mitochondria. Although our patient did also show a mild reduction in complex $\mathrm{V}$ activity, overall the changes were not specific for any respiratory chain disorder. The increased mitochondrial membrane cholesterol could lead to mitochondrial cytopathy in skeletal muscle in late-state disease in human patients. Targeted sequencing of mtDNA mutations, biomarkers such as plasma 3-methyl glutaconic acid, lactate, creatine kinase and neuroimaging were not supportive of the co-existence of a primary mitochondrial disease leading us to hypothesize that the mitochondrial abnormalities in NPC may be secondary.

The abnormal lysosomes seen on the skin biopsy have been previously described ${ }^{10}$. Considering the time to culture fibroblasts for cholesterol esterification studies and filipin staining and the fact that access to these tests may be difficult to obtain in some jurisdictions, the histopathology on skin biopsy should not be overlooked in patients suspected of NPC.

This is the first reported case of NPC in a Canadian Stoney Nakoda Nation child with a new reported mutation causing NPC. The biological parents declared that they were nonconsanguineous although a homozygous mutation was found indicating a possible early founder effect. The case illustrates the importance of considering lysosomal storage diseases in Aboriginal populations, the utility of examination of the skin using electron microscopy for storage diseases ${ }^{11}$, the importance of biochemical testing in situations where DNA testing identifies previously unclassified variants in small populations that may not be traditionally included in mutation databases. 


\section{REFERENCES}

1. Wraith JE, Guffon N, Rohrbach M, et al. Natural history of Niemann-Pick disease type $\mathrm{C}$ in a multicentre observational retrospective cohort study. Mol Genet Metab. 2009 Nov;98(3): 250-4.

2. Heron B, Ogier H. Niemann-Pick type C disease: clinical presentations in pediatric patients. Arch Pediatr. 2010;17 Suppl 2:S45-9.

3. Vanier MT. Niemann-Pick disease type C. Orphanet J Rare Dis. 2010;5:16.

4. Miller WL, Bose HS. Early steps in steroidogenesis: intracellular cholesterol trafficking. J Lipid Res. 2011;52(12):2111-35.

5. Harzer K, Kustermann-Kuhn B. Quantified increases of cholesterol, total lipid and globotriaosylceramide in filipin-positive Niemann-Pick type C fibroblasts. Clin Chim Acta. 2001;305(12):65-73.

6. Nicholson AG, Florio R, Hansell DM, et al. Pulmonary involvement by Niemann-Pick disease. A report of six cases. Histopathology. 2006;48(5):596-603.
7. Neville BG, Lake BD, Stephens R, Sanders MD. A neurovisceral storage disease with vertical supranuclear ophthalmoplegia, and its relationship to Niemann-Pick disease. A report of nine patients. Brain. 1973;96(1):97-120.

8. Mukherjee S, Maxfield FR. Lipid and cholesterol trafficking in NPC. Biochim Biophys Acta. 2004;1685(1-3):28-37.

9. Yu W, Gong JS, Ko M, Garver WS, Yanagisawa K, Michikawa M. Altered cholesterol metabolism in Niemann-Pick type $\mathrm{C} 1$ mouse brains affects mitochondrial function. J Biol Chem. 2005 Mar 25;280(12):11731-9.

10. Boustany RN, Kaye E, Alroy J. Ultrastructural findings in skin from patients with Niemann-Pick disease, type C. Pediatr Neurol. 1990;6(3):177-83

11. Prasad A, Kaye EM, Alroy J. Electron microscopic examination of skin biopsy is a cost-effective tool in the diagnosis of lysosomal storage diseases. J Child Neurol. 1996;11(4):301-8. 